\title{
INTEGRABLE MODULES FOR TWISTED TOROIDAL EXTENDED AFFINE LIE ALGEBRAS
}

\author{
S. ESWARA RAO, SACHIN S. SHARMA AND PUNITA BATRA
}

\begin{abstract}
In this paper we classify the irreducible integrable modules for the twisted toroidal extended affine Lie algebras (twisted toroidal EALA, in short) with finite dimensional weight spaces when the finite dimensional center acts non-trivially. Using an automorphism we reduce to the case where $K_{0}$ acts non-trivially and $K_{i}, 1 \leq i \leq n$ act trivially. Twisted toroidal EALA has natural triangular decomposition and we prove that any irreducible integrable module of it with finite dimensional weight spaces is a highest weight module with respect to the above triangular decomposition. The highest weight space is an irreducible module for the zeroth component of the twisted toroidal EALA. We then describe the highest weight space in detail.
\end{abstract}

MSC: $17 \mathrm{~B} 67,17 \mathrm{~B} 66$

KEY WORDS: Divergence zero vector fields, Lie torus, Extended affine Lie algebras.

\section{INTRODUCTION}

Extended affine Lie algebras (EALAs, for short) are natural generalization of affine Kac-Moody algebras; finite dimensional simple Lie algebra, extended toroidal Lie algebras are examples of EALAs. They first appeared in the work of Saito and Slodowy on elliptic singularities and in the paper of physicists Høegh and Torresani in 1990. Later mathematicians like Allison, 
Azam, Berman, Gao, Pianzola, Neher and Yoshii systematically developed the theory of EALAs (see [1, 21, 24], and references therein). Unlike affine Kac-Moody algebras, EALAs may have infinite dimensional centers. So representation theory of EALA's is still in progress.

Let $L(\stackrel{\circ}{\mathfrak{g}})=\stackrel{\circ}{\mathfrak{g}} \otimes A_{n}$ is a loop algebra, where $\stackrel{\circ}{\mathfrak{g}}$ is a finite dimensional simple Lie algebra over $\mathbb{C}$ and $A_{n}$ is a commutative Laurent polynomial ring of $n$ variables. Let $L(\mathfrak{g}) \oplus \Omega_{A_{n}} / d A_{n}$ be universal central extension of $L(\mathfrak{g})$ (note that the center is infinite dimensional for $n \geq 2$ ). These are called toroidal Lie algebras (TLA, in short) and they have been extensively studied [11, 13]. Let $\operatorname{Der}\left(A_{n}\right)$ be the Lie algebra of darivations on $A_{n}$ and consider the full toroidal Lie algebra (FTLA, in short) $L(\mathfrak{g}) \oplus \Omega_{A_{n}} / d A_{n} \oplus \operatorname{Der}\left(A_{n}\right)$. Representation of FTLA also have been studied [14]. Neither TLA nor FTLA are EALA as they do not admit non-degenerate symmetric invariant bilinear form. To rectify this one considers subalgebra $\mathcal{S}_{n}$ of $\operatorname{Der}\left(A_{n}\right)$ consisting of divergence zero vector fields (see Section 2 for more details). Then the Lie algebra $\tau_{\text {div }}=L(\mathfrak{g}) \oplus \Omega_{A_{n}} / d A_{n} \oplus \mathcal{S}_{n}$ is called toroidal extended affine Lie algebras. These are in fact EALAs. In [3] Billig constructed irreducible representation of toroidal EALAs using vertex operator algebras. In [4] Billig and Lau constructed irreducible modules for twisted toroidal EALAs. In [9, 10], full classification of irreducible integrable modules with finite dimensional weight spaces of toroidal EALA for $n=2$ was obtained. In this paper we assume $n \geq 3$ and classify irreducible integrable modules for toroidal EALAs where center acts non-trivially (see definition in Section 2). Actually we consider more general EALAs. We consider commutating 
INTEGRABLE MODULES FOR TWISTED TOROIDAL EXTENDED AFFINE LIE ALGEBRAS

finite order automorphisms $\sigma_{0}, \ldots, \sigma_{n}$ of $\stackrel{\circ}{\mathfrak{g}}$ and consider the multi-loop algebra $\bigoplus_{\left(k_{0}, k\right) \in \mathbb{Z}^{n+1}} \stackrel{\circ}{\mathfrak{g}}\left(\bar{k}_{0}, \bar{k}\right) t_{0}^{k_{0}} t^{k}$. We assume that multi-loop algebra is a Lie torus (see Section 2 for definition). This is not a very serious assumption as it is well known that centerless cores of almost all EALAs are Lie tori [1]. We then consider universal central extension of multi-loop algebra and add $\mathcal{S}_{n+1}$ the Lie algebra consisting of divergence zero vector fields on $A_{n+1}$ (see Section 2 for details). The resulting Lie algebra is EALA called as twised toroidal EALA, and we denote it by $\mathcal{T}$. The aim of this paper is to classify all irreducible integrable representation of twisted toroidal EALAs with finite dimensional weight spaces where center acts non-trivially.

The proof of our classification problem runs parallel to [2] where they consider full twisted TLA. Our twisted toroidal EALA is a proper subalgebra of full twisted EALA. Since we working with smaller algebra some of the results used in [2] won't work here. So we use differnt techniques; notably results from [5] and [15] are very special for divergence zero vector fields.

We now explain our plan of classification result in more detail. In Section 2 we start with basic definitions which leads up to the definition of twisted toroidal EALA $\mathcal{T}$. In Section 3 we define root space decomposition and using it define a natural triangular decomposition $\mathcal{T}=\mathcal{T}^{-} \oplus \mathcal{T}^{0} \oplus \mathcal{T}^{+}$. We fix an irreducible integrable module $V$ of $\mathcal{T}$ with finite dimensional weight spaces where $K_{0}$ acts non-trivially and $K_{i}$ act trivially for $1 \leq i \leq n$. This assumption is due to the fact that group $G L(n+1, \mathbb{Z})$ acts as automorphism on FTLA and leaves $\tau_{\text {div }}$ invariant. Then using earlier work of [2] it follows that the space $M=\left\{v \in V \mid \mathcal{T}^{+} v=0\right\}$ is a non-zero $\mathcal{T}^{0}$ irreducible module. The rest of the paper revolves around $M$ as we try to decode the structure of 
$M$. The $\mathcal{T}^{0}$-module $M$ has natural $\mathbb{Z}^{n}$ gradation and using integrality and Weyl group invariance of weights of $V$, we prove the dimensions of graded pieces of $M$ are uniformly bounded. Using this we prove that $\Omega_{A(m)} / d_{A(m)}$ acts trivially on $M$ (Propostion 3.8). Then we prove proposition 3.10 which encodes the action subspace $\sum_{r \in \Gamma} \mathbb{C} t^{r} K_{0} \oplus \sum_{r \in \Gamma} \mathbb{C} t^{r} d_{0}$ of $\mathcal{T}^{0}$. We would like to emphasize the fact unlike 2] where the corresponding results come by earlier works of authors, we need completely different techinque to prove Proposition 3.10, For its proof hinges on results from [15] (Theorem 3.9] in our paper) and [8]. By Propsition 3.10 it follows that $M \cong M^{1} \otimes A(m)$ for some space $M^{1}$. Then for some suitable subspace $W$ of $M$, we consider $\widetilde{V}=M / W$. Here $W$ and $\widetilde{V}$ are not $\mathcal{T}^{0}$ - modules but modules for some suitable Lie algebra $\mathcal{I}$ (see Sections 3 and 4 for details ). It follows from the work of [2] that $\widetilde{V}$ is completely reducible $\mathcal{I}$-module with isomorphic irreducible components. In Section 4, using results of [22] and [5] we classify all the finite dimensional irreducible modules for $\mathcal{I}$. Finally in Section 5 by going up procedure we define module for $\mathcal{T}^{0}$, which contains an isomorphic copy of $M$ and we conclude with the final result Theorem 5.1 ,

\section{2 .}

Let $A_{n+1}=\mathbb{C}\left[t_{0}^{ \pm 1}, \cdots, t_{n}^{ \pm 1}\right]$ be a Laurent polynomial ring in $n+1$ variables. $L(\stackrel{\circ}{\mathfrak{g}})=\stackrel{\circ}{\mathfrak{g}} \otimes A_{n+1}$ be the corresponding loop algebra, $\stackrel{\circ}{\mathfrak{g}}$ is a finite dimensional simple Lie algebra over $\mathbb{C}$ with a Cartan subalgebra $\stackrel{\circ}{\mathfrak{h}}$. Let $\Omega_{A_{n+1}}$ be a vector space spanned by the symbols $t_{0}^{k_{0}} t^{k} K_{i}, 0 \leq i \leq n, k_{0} \in \mathbb{Z}, k \in \mathbb{Z}^{n}$. Let $d A_{n+1}$ be the subspace spanned by $\sum_{i=0}^{n} k_{i} t_{0}^{k_{0}} t^{k} K_{i}$. It is well known that 
INTEGRABLE MODULES FOR TWISTED TOROIDAL EXTENDED AFFINE LIE ALGEBRAS $\tilde{L}(\mathfrak{g})=L(\mathfrak{g}) \oplus \Omega_{A_{n+1}} / d A_{n+1}$ is the universal central extension of $L(\mathfrak{g})$ with the following brackets:

$$
\left[X\left(k_{0}, k\right), Y\left(l_{0}, l\right)\right]=[X, Y]\left(l_{0}+k_{0}, l+k\right)+(X \mid Y) \sum_{i=0}^{n} k_{i} t_{0}^{l_{0}+k_{0}} t^{l+k} K_{i},
$$

where $X\left(k_{0}, k\right)=X \otimes t_{0}^{k_{0}} t^{k}$. Recall the Lie algebra of derivations of $A_{n+1}$, $\operatorname{Der}\left(A_{n+1}\right)$ with basis $\left\{d_{i}, t_{0}^{r_{0}} t^{r} d_{i} \mid 0 \leq i \leq n, 0 \neq\left(r_{0}, r\right) \in \mathbb{Z}^{n+1}\right\} . \operatorname{Der}\left(A_{n+1}\right)$ acts on $\Omega_{A_{n+1}} / d A_{n+1}$ by

$$
t_{0}^{p_{0}} t^{p} d_{a}\left(t_{0}^{q_{0}} t^{q} K_{b}\right)=q_{a} t_{0}^{p_{0}+q_{0}} t^{p+q} K_{b}+\delta_{a b} \sum_{c=0}^{n} p_{c} t_{0}^{p_{0}+q_{0}} t^{p+q} K_{c} .
$$

There are two non-trivial 2-cocycle of $\operatorname{Der}\left(A_{n+1}\right)$ with values in $\Omega_{A_{n+1}} / d A_{n+1}$ :

$$
\begin{aligned}
& \phi_{1}\left(t_{0}^{p_{0}} t^{p} d_{a}, t_{0}^{q_{0}} t^{q} d_{b}\right)=-q_{a} p_{b} \sum_{c=0}^{n} p_{c} t_{0}^{p_{0}+q_{0}} t^{p+q} K_{c} \\
& \phi_{1}\left(t_{0}^{p_{0}} t^{p} d_{a}, t_{0}^{q_{0}} t^{q} d_{b}\right)=-p_{a} q_{b} \sum_{c=0}^{n} p_{c} t_{0}^{p_{0}+q_{0}} t^{p+q} K_{c} . \text { Let } \phi \text { be any linear }
\end{aligned}
$$

combination of $\phi_{1}$ and $\phi_{2}$. Then $\tau=L(\stackrel{\circ}{\mathfrak{g}}) \oplus \Omega_{A_{n+1}} / d A_{n+1} \oplus \operatorname{Der}\left(A_{n+1}\right)$

is a Lie algebra with the following brackets and called as full toroidal Lie algebra (FTLA, for short):

$$
\begin{aligned}
{\left[t_{0}^{p_{0}} t^{p} d_{a}, X\left(q_{0}, q\right)\right] } & =q_{a} X\left(p_{0}+q_{0}, p+q\right) \\
{\left[t_{0}^{p_{0}} t^{p} d_{a}, t_{0}^{q_{0}} t^{q} K_{b}\right] } & =q_{a} t_{0}^{p_{0}+q_{0}} t^{p+q} K_{b}+\delta_{a b} \sum_{c=0}^{n} p_{c} t_{0}^{p_{0}+q_{0}} t^{p+q} K_{c} \\
{\left[t_{0}^{p_{0}} t^{p} d_{a}, t_{0}^{q_{0}} t^{q} d_{b}\right] } & =q_{a} t_{0}^{p_{0}+q_{0}} t^{p+q}-p_{b} t_{0}^{p_{0}+q_{0}} t^{p+q}+\phi\left(t_{0}^{p_{0}} t^{p} d_{a}, t_{0}^{q_{0}} t^{q} d_{b}\right)
\end{aligned}
$$

Now consider the subalgebra of divergence zero vector fields $\mathcal{S}_{n+1}$ of $\operatorname{Der}\left(A_{n+1}\right)$.

One can define $\mathcal{S}_{n+1}=\left\{D(u, r) \mid(u \mid r)=0, u \in \mathbb{C}^{n+1}, r \in \mathbb{Z}^{n+1}\right\}$.

Now consider the subalgebra $\tau_{\text {div }}=L(\mathfrak{g}) \oplus \Omega_{A_{n+1}} / d A_{n+1} \oplus \mathcal{S}_{n+1}$ of $\tau$. It is well known that unlike $\tau, \tau_{\text {div }}$ possesses a non-degenerate symmetric, invariant bilinear form and is called as toroidal extended affine Lie algebra. 
The form is defined as follows:

$$
\begin{aligned}
& \left(X \otimes t^{r} \mid Y \otimes t^{s}\right)=\delta_{r,-s}(X \mid Y), X, Y \in \stackrel{\circ}{\mathfrak{g}}, r, s \in \mathbb{Z}^{n+1} ; \\
& \left(\sum_{c=0}^{n} a_{c} t^{r} d_{c} \mid t^{s} K_{d}\right)=\delta_{r,-s} a_{d} .
\end{aligned}
$$

All other brackets of bilinear form are zero. Now let $\mathfrak{g}_{1}$ be any arbitrary finite dimensional simple Lie algebra $\mathbb{C}$ with a Cartan subalgebra $\mathfrak{h}_{1}$. Let $\Delta\left(\mathfrak{g}_{1}, \mathfrak{h}_{1}\right)=\operatorname{supp}_{\mathfrak{h}_{1}}\left(\mathfrak{g}_{1}\right)$. Then $\Delta_{1}^{\times}=\Delta^{\times}\left(\mathfrak{g}_{1}, \mathfrak{h}_{1}\right)=\Delta\left(\mathfrak{g}_{1}, \mathfrak{h}_{1}\right) \backslash\{0\}$ is an irreducible reduced finite root system with at most two root lengths. Let $\Delta_{1, \mathrm{sh}}^{\times}$denote the non zero short roots of $\Delta_{1}$. Define

$$
\Delta_{1, \text { en }}^{\times}= \begin{cases}\Delta_{1}^{\times} \cup 2 \Delta_{1, \text { sh }}^{\times} & \text {if } \Delta_{1}^{\times}=B_{l} \text { type } \\ \Delta_{1}^{\times} & \text {otherwise. }\end{cases}
$$

We need the following definition:

Definition 2.1. A finite dimensional $\mathfrak{g}_{1}$-module $V$ is said to satisfy condition $(M)$ if $V$ is irreducible with dimension greater than 1 and weights of $V$ relative to $\mathfrak{h}_{1}$ are contained in $\Delta_{1, \mathrm{en}}^{\times}$.

Now recall that $\stackrel{\circ}{\mathfrak{g}}$ is a finite dimensional simple Lie algebra with a Cartan subalgebra $\stackrel{\circ}{\mathfrak{h}}$ and let $\sigma_{0}, \sigma_{1}, \cdots, \sigma_{n}$ be the commuting automorphism of $\stackrel{\circ}{\mathfrak{g}}$ of order $m_{0}, m_{1}, \cdots, m_{n}$ respectively. Let $m=\left(m_{1}, \ldots, m_{n}\right) \in \mathbb{Z}^{n}$. Define $\Gamma_{0}=m_{0} \mathbb{Z}$ and $\Gamma=m_{1} \mathbb{Z} \oplus \cdots \oplus m_{n} \mathbb{Z}$. Let $\Lambda_{0}:=\mathbb{Z} / \Gamma_{0}$ and $\Lambda:=\mathbb{Z}^{n} / \Gamma$. Then we have $\stackrel{\circ}{\mathfrak{g}}=\bigoplus_{\left(\bar{k}_{0}, \bar{k}\right)} \stackrel{\circ}{\mathfrak{g}}\left(\bar{k}_{0}, \bar{k}\right)$, where $\left(\bar{k}_{0}, \bar{k}\right)=\left(\bar{k}_{0}, \bar{k}_{1} \ldots, \bar{k}_{n}\right) \in \Gamma_{0} \oplus \Gamma$, and $\stackrel{\circ}{\mathfrak{g}}\left(\bar{k}_{0}, \bar{k}\right)=\left\{X \in \stackrel{\circ}{\mathfrak{g}} \mid \sigma_{i}(X)=\xi_{i}^{k_{i}} X, 0 \leq i \leq n\right\}$, where $\xi_{i}$ are $m_{i}$-th primitive root of unity for $i=0 \ldots, n$. 
Definition 2.2. A multiloop algebra $\bigoplus_{\left(k_{0}, k\right) \in \mathbb{Z}^{n+1}} \mathfrak{g}\left(\bar{k}_{0}, \bar{k}\right) t_{0}^{k_{0}} t^{k}$ is called a Lie torus $L T$ if

(1) $\stackrel{\circ}{\mathfrak{g}}(\overline{0}, \overline{0})$ is a finite dimensional simple Lie algebra.

(2) For $\left(\bar{k}_{0}, \bar{k}\right) \neq(0,0)$ and $\mathfrak{g}\left(\bar{k}_{0}, \bar{k}\right) \neq 0, \mathfrak{g}\left(\bar{k}_{0}, \bar{k}\right) \cong U\left(\bar{k}_{0}, \bar{k}\right) \oplus W\left(\bar{k}_{0}, \bar{k}\right)$, where $U\left(\bar{k}_{0}, \bar{k}\right)$ is trivial as $\mathfrak{g}(\overline{0}, \overline{0})$-module and either $W\left(\bar{k}_{0}, \bar{k}\right)$ is zero or satisfy the condition (M).

(3) The order of the group generated by $\sigma_{i}, 0 \leq i \leq n$ is equal to the product of orders of each $\sigma_{i}$, for $0 \leq i \leq n$.

Let $\stackrel{\circ}{\mathfrak{h}}(0)$ denote a Cartan subalgebra of $\stackrel{\circ}{\mathfrak{g}}(\overline{0}, \overline{0})$. Then by [20] Lemma 3.1.3, $\stackrel{\circ}{\mathfrak{h}}(0)$ is ad-diagonalizable on $\stackrel{\circ}{\mathfrak{g}}$ and $\Delta^{\times}=\Delta^{\times}(\stackrel{\circ}{\mathfrak{g}}, \mathfrak{h}(0))$ is an irreducible finite root system in $\stackrel{\circ}{\mathfrak{h}}(0)$ (Proposition 3.3.5, [20]). Let $\Delta_{0}:=\Delta(\mathfrak{g}(\overline{0}, \overline{0}), \mathfrak{h}(0))$ One of the main properties of Lie tori is that $\Delta:=\Delta(\mathfrak{g}, \stackrel{\circ}{\mathfrak{h}}(0))=\Delta_{0 \text {,en }}$ (Proposition 3.2.5, [1]). Let $A(m)=\mathbb{C}\left[t_{1}^{ \pm m_{1}}, \ldots, t_{n}^{ \pm m_{n}}\right]$ and $A\left(m_{0}, m\right)=$ $\mathbb{C}\left[t_{0}^{ \pm m_{0}}, t_{1}^{ \pm m_{1}}, \ldots, t_{n}^{ \pm m_{n}}\right]$ and $\mathcal{S}_{n+1}\left(m_{0}, m\right)=\left\{D(u, r) \mid(u \mid r)=0, u \in \mathbb{C}^{n+1}, r \in\right.$ $\left.\Gamma_{0} \oplus \Gamma\right\}$. The Lie algebra $\mathcal{T}=L T \bigoplus \Omega_{A\left(m_{0}, m\right)} / d_{A\left(m_{0}, m\right)} \bigoplus \mathcal{S}_{n+1}\left(m_{0}, m\right)$ is called twisted toroidal extended affine Lie algebra . The purpose of this paper is to classify the irreducible integrable modules of twisted toroidal EALAs. 
Automorphism: Let $B=\left(b_{i j}\right) \in G L(n+1, Z)$ and $B^{-1}=\left(c_{i j}\right)$, then the following is an automorphism $\phi_{B}$ on $\tau$ by:

$$
\begin{aligned}
\phi_{B}\left(X \otimes t^{\left(k_{0}, k\right)}\right) & =X \otimes t^{\left(k_{0}, k\right) B^{t}}, \\
\phi_{B}\left(t^{\left(k_{0}, k\right)} K_{i}\right) & =\sum_{p=0}^{n} b_{i p} t^{\left(k_{0}, k\right) B^{t}}, \\
\phi_{B}\left(t^{\left(k_{0}, K\right)} d_{j}\right) & =\sum_{p=0}^{n} c_{j p} t^{\left(k_{0}, k\right) B^{t}} .
\end{aligned}
$$

It is easy to check that $\phi_{B}$ is an automorphism on $\tau$. Now as for $u \in$ $\mathbb{C}^{n+1}, r \in \mathbb{Z}^{n+1}$ such that $(u \mid r)=0, \phi_{B} D(u, r)=D\left(u B^{-1}, r B^{t}\right)$ and as $\left(u B^{-1} \mid r B^{t}\right)=\left(u \mid r B^{t} B^{-1 t}\right)=0$. So $\mathcal{S}_{n+1}$ is invariant under $\phi_{B}$. Hence $\phi_{B}$ leaves $\tau_{\text {div }}$ invariant. So up to an automorphism we can assume that the central element $K_{0}$ acts as $C_{0} \in \mathbb{Z}_{>0}$ and $K_{i}$ 's as 0 for $1 \leq i \leq n$.

\section{3.}

In this section we will do root decomposition of $\mathcal{T}$. Let $\mathcal{H}=\stackrel{\circ}{\mathfrak{h}}(0) \oplus$ $\sum_{i=0}^{n} \mathbb{C} K_{i} \oplus \sum_{i=0}^{n} \mathbb{C} d_{i}$ will be our Cartan subalgebra for the root decomposition of $\mathcal{T}$. Define $\delta_{i}, \omega_{i} \in \mathcal{H}^{*}$ for $0 \leq i \leq n$ by $\delta_{i}(\grave{\mathfrak{h}}(0))=0, \delta_{i}\left(K_{j}\right)=0, \delta_{i}\left(d_{j}\right)=$ $\delta_{i j}$ and $\omega_{i}(\grave{h}(0))=0, \omega_{i}\left(K_{j}\right)=\delta_{i j}, \omega_{i}\left(d_{j}\right)=0$. Let us denote $\delta_{k}=\sum_{i=1}^{n} k_{i} \delta_{i}$ for $k \in \mathbb{Z}^{n}$. Define $\stackrel{\circ}{\mathfrak{g}}\left(\bar{k}_{0}, \bar{k}, \alpha\right):=\left\{x \in \stackrel{\circ}{\mathfrak{g}}\left(\bar{k}_{0}, \bar{k}\right) \mid[h, x]=\alpha(h) x, \forall h \in \mathfrak{h}(0)\right\}$ Then we have $\mathcal{T}=\bigoplus_{\beta \in \Delta} \mathcal{T}_{\beta}$, where $\Delta \subseteq\left\{\alpha+k_{0} \delta_{0}+\delta_{k} \mid \alpha \in \Delta_{0, \text { en }}, k_{0} \in \mathbb{Z}, k \in\right.$ $\left.\mathbb{Z}^{n}\right\}$. We have $\mathcal{T}_{\alpha+k_{0} \delta_{0}+\delta_{k}}=\stackrel{\circ}{\mathfrak{g}}\left(\bar{k}_{0}, \bar{k}, \alpha\right) \otimes t_{0}^{k_{0}} t^{k}$ for $\alpha \neq 0$, and $\mathcal{T}_{k_{0} \delta_{0}+\delta_{k}}=$ $\stackrel{\circ}{\mathfrak{g}}\left(\bar{k}_{0}, \bar{k}, 0\right) \otimes t_{0}^{k_{0}} t^{k} \oplus \bigoplus_{i=0}^{n} \mathbb{C} t_{0}^{k_{0}} t^{k} K_{i} \oplus \bigoplus_{i=0}^{n} \mathbb{C} t_{0}^{k_{0}} t^{k} d_{i}$ for $k_{0} \delta_{0}+\delta_{k} \neq 0$ and $\mathcal{T}_{0}=\mathcal{H}$

In order to define a non-degenerate form on $\mathcal{H}^{*}$ we first extend $\alpha \in \stackrel{\circ}{\mathfrak{h}}(0)^{*}$ to $\mathcal{H}$ by defining $\alpha\left(K_{i}\right)=\alpha\left(d_{i}\right)=0, \forall 0 \leq i \leq n$. Then $\left(\mathfrak{h}(0) \mid K_{i}\right)=$ 
INTEGRABLE MODULES FOR TWISTED TOROIDAL EXTENDED AFFINE LIE ALGEBRA9 $\left(\stackrel{\circ}{\mathfrak{h}}(0) \mid d_{i}\right),\left(\delta_{k}+\delta_{k_{0}} \mid \delta_{l}+\delta_{l_{0}}\right)=\left(\omega_{i} \mid \omega_{j}\right)=\left(\delta_{k}, \delta_{l}\right)=0$ and $\left(\delta_{i} \mid \omega_{j}\right)=\delta_{i j}$, and form on $\stackrel{\circ}{\mathfrak{h}}(0)$ is restriction of the form of $\stackrel{\circ}{\mathfrak{g}}$. It is easy to see that this form is non-degenerate on $\mathcal{H}^{*}$. A root $\beta=\alpha+k_{0} \delta_{0}+\delta_{k}$ is called real root if $\alpha \neq 0$. Let $\Delta^{\text {re }}$ denote the set of all real roots, and $\beta^{\vee}=\alpha^{\vee}+\frac{2}{(\alpha \mid \alpha)} \sum_{i=0}^{n} k_{i} K_{i}$ is coroot of $\beta$, where $\alpha^{\vee}$ is co-root of $\alpha \in \Delta_{0 \text {,en }}$. Then $\beta\left(\beta^{\vee}\right)=\alpha\left(\alpha^{\vee}\right)=2$. For any real root $\gamma$ of $\mathcal{T}$, define the reflection $r_{\gamma}$ on $\mathcal{H}^{*}$ by $r_{\gamma}(\lambda)=\lambda-\lambda\left(\gamma^{\vee}\right) \gamma$ for $\lambda \in \mathcal{H}^{*}$. Let Weyl group $W$ of $\mathcal{T}$ be the group generated by $r_{\gamma}, \forall \gamma \in \Delta^{\text {re }}$. Now we define the category of integrable modules for $\mathcal{T}$.

Definition 3.1. A $\mathcal{T}$-module $V$ is called integrable if

(1) $V=\bigoplus_{\lambda \in \mathcal{H}^{*}} V_{\lambda}$, where $V_{\lambda}=\{v \in V \mid h . v=\lambda(h) v \forall h \in \mathcal{H}\}$ and $\operatorname{dim}\left(V_{\lambda}\right)<\infty$.

(2) All the real root vectors act locally nilpotently on $V$, i.e., $\stackrel{\circ}{\mathfrak{g}}\left(\bar{k}_{0}, \bar{k}, \alpha\right) \otimes$ $t_{0}^{k_{0}} t^{k}$ acts locally nilpotently on $V$ for all $0 \neq \alpha \in \Delta_{0, \text { en }}$.

We have the following :

Proposition 3.2. Let $V$ be an irreducible integrable module for $\mathcal{T}$. Then

(1) $P(V)=\left\{\gamma \in \mathcal{H}^{*} \mid V_{\gamma} \neq 0\right\}$ is $W$ - invariant.

(2) $\operatorname{dim}\left(V_{\gamma}\right)=\operatorname{dim}\left(V_{w \gamma}\right), \forall w \in W$.

(3) If $\lambda \in P(V)$ and $\gamma \in \Delta^{\mathrm{re}}$, then $\lambda\left(\gamma^{\vee}\right) \in \mathbb{Z}$.

(4) If $\lambda \in P(V)$ and $\gamma \in \Delta^{\mathrm{re}}$, and $\lambda\left(\gamma^{\vee}\right)>0$, then $\lambda-\gamma \in P(V)$.

(5) For $\lambda \in P(V), \lambda\left(K_{i}\right)$ is an integer independent of $\lambda$.

3.1. Now rest of this paper we will work with irreducible integrable representation $V$ of $\mathcal{T}$ with central element $K_{0}$ acts as $C_{0} \in \mathbb{Z}_{>0}$ and rest of $K_{i}$ 's act trivially for $1 \leq i \leq n$. We now define an order on $\mathcal{H}^{*}$. Let for $\lambda \in \mathcal{H}^{*}$, 
$\lambda^{\prime}$ denote the restriction of $\lambda$ to $\stackrel{\circ}{\mathfrak{h}}(0)^{*}$ and for a given $\lambda^{\prime} \in \stackrel{\circ}{\mathfrak{h}}(0)^{*}$, extend it to $\mathcal{H}^{*}$ by defining $\lambda^{\prime}\left(K_{i}\right)=0$ and $\lambda^{\prime}\left(d_{i}\right)=0$ for $0 \leq i \leq n$. Then we have unique expression for $\lambda=\lambda^{\prime}+\sum_{i=0}^{n} \lambda\left(K_{i}\right) \omega_{i}+\sum_{i=0}^{n} \lambda\left(d_{i}\right) \delta_{i}$ and for $\lambda \in P(V)$ we have $\lambda=\lambda^{\prime}+\lambda\left(K_{0}\right) \omega_{0}+\lambda\left(d_{0}\right) \delta_{0}+\sum_{i=1}^{n} \lambda\left(d_{i}\right) \delta_{i}$. So for $\lambda \in P(V)$, we write $\lambda=\bar{\lambda}+\sum_{i=i}^{n} \lambda\left(d_{i}\right) \delta_{i}$, where $\bar{\lambda}=\lambda^{\prime}+\lambda\left(K_{0}\right) \omega_{0}+\lambda\left(d_{0}\right) \delta_{0}$. Let $\beta_{0}$ be a maximal root in $\Delta_{0, \text { en }}$. Define $\alpha_{0}=-\beta_{0}+\delta_{0}$, which may not be a root $\mathcal{T}$. Let $\alpha_{1}, \ldots, \alpha_{q}$ be simple roots of $\Delta(\stackrel{\circ}{\mathfrak{g}}(\overline{0}, \overline{0}), \stackrel{\circ}{\mathfrak{h}}(0))$ and define the lattice $Q^{+}=\bigoplus_{i=0}^{q} \mathbb{N} \alpha_{i}$. Let for $\Lambda, \lambda \in \mathcal{H}^{*}$, we define an ordering on $\mathcal{H}$ by $\lambda \leq \Lambda$ if $\Lambda-\lambda \in Q^{+}$. It is easy to see that for $\Lambda \leq \lambda, \Lambda\left(d_{i}\right)=\lambda\left(d_{i}\right)$ for $1 \leq i \leq n$. 
Consider the natural triangular decomposition of $\mathcal{T}$ :

$$
\begin{aligned}
& L T^{+}=\bigoplus_{\alpha+k_{0} \delta_{0}>0, k \in \mathbb{Z}^{n}} \mathfrak{g}\left(\bar{k}_{0}, \bar{k}, \alpha\right) \otimes t_{0}^{k_{0}} t^{k} \\
& L T^{-}=\bigoplus_{\alpha+k_{0} \delta_{0}<0, k \in \mathbb{Z}^{n}} \mathfrak{g}\left(\bar{k}_{0}, \bar{k}, \alpha\right) \otimes t_{0}^{k_{0}} t^{k} \\
& L T^{0}=\bigoplus_{k \in \mathbb{Z}^{n}} \mathfrak{g}(\overline{0}, \bar{k}, 0) \otimes t^{k} \\
& \mathcal{S}_{n+1}^{+}\left(m_{0}, m\right)=\left\{D(u, r) \mid u \in \mathbb{C}^{n+1}, r \in \Gamma_{0} \oplus \Gamma,(u \mid r)=0, r_{0}>0\right\} ; \\
& \mathcal{S}_{n+1}^{-}\left(m_{0}, m\right)=\left\{D(u, r) \mid u \in \mathbb{C}^{n+1}, r \in \Gamma_{0} \oplus \Gamma,(u \mid r)=0, r_{0}<0\right\} ; \\
& \mathcal{S}_{n+1}^{0}\left(m_{0}, m\right)=\left\{D(u, r) \mid u \in \mathbb{C}^{n+1}, r \in \Gamma_{0} \oplus \Gamma,(u \mid r)=0, r_{0}=0\right\} ; \\
& Z^{+}=\bigoplus_{\substack{0 \leq i \leq n \\
0<s_{0} \in \Gamma_{0}, s \in \Gamma}} \mathbb{C} t_{0}^{s_{0}} t^{s} K_{i} \\
& Z^{-}=\bigoplus_{\substack{0 \leq i \leq n \\
0>s_{0} \in \Gamma_{0}, s \in \Gamma}} \mathbb{C} t_{0}^{s_{0}} t^{s} K_{i} ; \\
& Z^{0}=\bigoplus_{0 \leq i \leq n, s \in \Gamma} \mathbb{C} t^{s} K_{i}
\end{aligned}
$$

Let $\mathcal{T}^{+}=L T^{+} \oplus Z^{+} \oplus \mathcal{S}_{n+1}^{+}\left(m_{0}, m\right), \mathcal{T}^{-}=L T^{-} \oplus Z^{-} \oplus \mathcal{S}_{n+1}^{-}\left(m_{0}, m\right)$ and $\mathcal{T}^{0}=L T^{0} \oplus Z^{0} \oplus \mathcal{S}_{n+1}^{0}\left(m_{0}, m\right)$. Then $\mathcal{T}=\mathcal{T}^{-} \oplus \mathcal{T}^{0} \oplus \mathcal{T}^{+}$is a trigular decomposition of $\mathcal{T}$. We have the following

Theorem 3.3. The space $M=\left\{v \in V \mid \mathcal{T}^{+} v=0\right\}$ is non-zero.

Proof. It will suffice to show the existence of $\Lambda \in P(V)$ such that $\Lambda+\beta+\delta_{k} \notin$ $P(V)$ for any root $\beta+\delta_{k} \in P(V)$ with $\beta>0$ and $k \in \mathbb{Z}^{n}$. The proof is similar to the proof of Theorem 5.2 of [2].

It follows that $M$ is a irreducible $\mathcal{T}^{0}$-module and $U\left(\mathcal{T}^{-}\right) M=V$. Note that $M$ is $\mathbb{Z}^{n}$-graded as $d_{i} \in \mathcal{S}_{n+1}^{0}\left(m_{0}, m\right)$. We identify $\mathcal{S}_{n+1}^{0}\left(m_{0}, m\right)$ with 
$\mathcal{S}_{n}(m) \oplus \sum_{r \in \Gamma} t^{r} d_{0}$, where $\mathcal{S}_{n}(m)=\left\{D(u, r): u \in \mathbb{C}^{n}, r \in \Gamma,(u \mid r)=0\right\}$. So we identify $\mathcal{T}^{0}$ with $L T^{0} \oplus \mathcal{S}_{n}(m) \oplus \sum_{r \in \Gamma} \mathbb{C} t^{r} d_{0} \oplus Z^{0}$.

Now we will prove that weight spaces of $M$ are uniformly bounded and $\left\{t^{s} K_{i} \mid s \in \Gamma, 1 \leq i \leq n\right\}$ acts trivially on $M$. For this let us fix an $i, 1 \leq i \leq n$ and consider the loop algebra $\stackrel{\circ}{\mathfrak{g}}(\overline{0}, \overline{0}) \otimes \mathbb{C}\left[t_{i}^{m_{i}}, t_{i}^{-m_{i}}\right] \oplus d_{i}$. Let $\theta$ be the highest root of $\stackrel{\circ}{\mathfrak{g}}(\overline{0}, \overline{0})$ and $\theta^{\vee}$ be the coroot. Now as $\stackrel{\circ}{\mathfrak{h}}(0)$ acts as scalar on $M$, let $\lambda \in \stackrel{\circ}{\mathfrak{h}}(0)^{*}$ such that $h(v)=\lambda(h) v, \forall h \in \stackrel{\circ}{\mathfrak{h}}(0), \forall v \in M$, here $\lambda$ is restriction

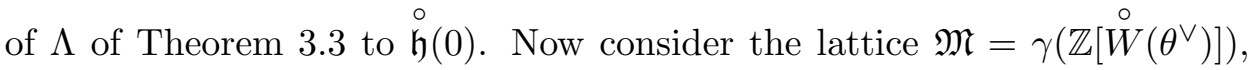
where $\stackrel{\circ}{W}$ denote the finite Weyl group of $\stackrel{\circ}{\mathfrak{g}}(\overline{0}, \overline{0})$ and $\gamma: \stackrel{\circ}{\mathfrak{h}}(0) \mapsto \stackrel{\circ}{\mathfrak{h}}(0)^{*}$ is an isomorphism. Consider the element $t_{i, h}(\lambda)=\lambda-\lambda(h) \delta_{i}$, where $h \in$ $\mathbb{Z}\left[\stackrel{\circ}{W}\left(\theta^{\vee}\right)\right]$. Note that $\lambda\left(K_{i}\right)=0$. Now as $t_{i, h_{1}} t_{i, h_{2}}=t_{i, h_{1}+h_{2}}$, we can identify the group generated by $\left\{t_{i, h}: h \in \mathbb{Z}\left[\stackrel{\circ}{W}\left(\theta^{\vee}\right)\right]\right\}$ with $\mathfrak{M}$. Let $r_{\lambda}=\min \{\lambda(h)$ : $\left.h \in \mathbb{Z}\left[\stackrel{\circ}{W}\left(\theta^{\vee}\right)\right], \lambda(h)>0\right\}$.

Lemma 3.4. Consider $\lambda+r \delta_{i}, r \in \mathbb{Z}$. Then there exists $w \in \mathfrak{M}$ such that $w\left(\lambda+r \delta_{i}\right)=\lambda+\bar{s} \delta_{i}$, for $0 \leq \bar{s}<r_{\lambda}$

Proof. See 2.4 of [7].

Now consider the Lie algebra $\stackrel{\widetilde{\circ}}{\mathfrak{h}}(A(m))=\stackrel{\circ}{\mathfrak{h}}(0) \otimes A(m) \bigoplus \Omega_{A(m)} / d(A(m))$. Let $\widetilde{W}$ be the Weyl group of the corresponding FTLA

$$
\stackrel{\circ}{\mathfrak{g}}(\overline{0}, \overline{0}) \otimes A(m) \bigoplus \Omega_{A(m)} / d(A(m)) \bigoplus \operatorname{Der}(A(m))
$$

As before consider the loop subalgebra $\stackrel{\circ}{\mathfrak{g}}(\overline{0}, \overline{0}) \otimes \mathbb{C}\left[t_{i}^{m_{i}}, t_{i}^{-m_{i}}\right] \oplus d_{i}$ of the FTLA. By the discussion above we have $t_{i, h} \lambda=\lambda-\lambda(h) \delta_{i}$ an element of $\widetilde{W}_{i} \subseteq \widetilde{W}$, where $\widetilde{W}_{i}$ denote the corresponding Weyl group of $\stackrel{\circ}{\mathfrak{g}}(\overline{0}, \overline{0}) \otimes \mathbb{C}\left[t_{i}^{m_{i}}, t_{i}^{-m_{i}}\right] \oplus d_{i}$. Then for $s_{i} \in \mathbb{Z}$ and $\lambda+s_{i} \delta_{i}=\lambda+k_{i} \delta_{i}+r_{i} m_{i} \delta_{i}$, where $s_{i}=k_{i}+r_{i} m_{i}$, 
INTEGRABLE MODULES FOR TWISTED TOROIDAL EXTENDED AFFINE LIE ALGEBRAS

$0 \leq k_{i}<m_{i}$. Then by the Lemma 3.4 we get $w_{i} \in \widetilde{W}$ such that $w_{i}\left(\lambda+s_{i} \delta_{i}\right)=$ $\lambda+k_{i} \delta_{i}+\bar{s}_{i} \delta_{i}$, where $0 \leq k_{i}<m_{i}$ and $0 \leq \bar{s}_{i}<r_{\lambda}$.

Corollary 3.5. Let $\delta_{r}=\sum_{i=1}^{n} r_{i} \delta_{i}$, where $r=\left(r_{1}, \ldots, r_{n}\right) \in \mathbb{Z}^{n}$. Let $r_{i}=k_{i}+s_{i} m_{i}, 0 \leq k_{i}<m_{i}$. Then there exists $w \in \widetilde{W}$ such that $w\left(\lambda+\delta_{r}\right)=$ $\delta_{k}+\sum_{i=1}^{n} \bar{s}_{i} m_{i} \delta_{i}+\lambda$, where $0 \leq \bar{s}_{i}<r_{\lambda}$.

Now consider $M=\bigoplus_{k \in \mathbb{Z}^{n}} M_{k}$. Let $M^{\prime}=\left\{M_{l}: l \in \mathbb{Z}^{n}, l_{i}=k_{i}+m_{i} \bar{s}_{i}, 0 \leq\right.$ $\left.k_{i}<m_{i}, 0 \leq \bar{s}_{i}<r_{\lambda}, 1 \leq i \leq n\right\}$. Then clearly $M^{\prime}$ is a finite set. Let $N=\operatorname{Max} \operatorname{dim}\left\{T_{s}: T_{s} \in M^{\prime}\right\}$.

Proposition 3.6. The dimensions of the weight spaces of $M$ are uniformly bounded by $N$.

Proof. Follows from Corollary 3.5.

Proposition 3.7. There are only finitely many $\stackrel{\widetilde{b}}{\mathfrak{h}}(A(m))$-submodules of $M$.

Proof. Any such module $S$ is generated by vectors of $M$, which can be taken from the generators of $M^{\prime}$ by corollary 3.5 .

$\widetilde{0}$

Now as number of $\stackrel{\circ}{\mathfrak{h}}(A(m))$-modules are finite consider minimal such module say $S_{\text {min }}$. Clearly $S_{\text {min }}$ is an irreducible $\tilde{\stackrel{h}{\mathfrak{h}}}(A(m))$-module and consider $\left(U(\mathfrak{g}(\overline{0}, \overline{0})) \oplus \Omega_{A(m)} / d_{A(m)}\right) S_{\text {min }}$ which is an integrable module with top part irreducible as $\tilde{\mathfrak{h}}(A(m))$-module. Now consider the irreducible quotient of above module. Notice that top part goes injectively to the quotient. It follows by [11] Remark 5.5 that $\Omega_{A(m)} / d_{A(m)}$ acts trivially on $S_{\min }$. Now consider $\bar{M}=\left\{v \in M \mid \Omega_{A(m)} / d_{A(m)} v=0\right\}$. Then it is easy to see that $\bar{M}$ is a $\mathcal{T}^{0}$-module. But this forces $M=\bar{M}$ by irreducibility of $M$ as $\mathcal{T}^{0}$-module and we have the following: 
Proposition 3.8. $\Omega_{A(m)} / d_{A(m)}$ acts trivially on $M$.

Using above we further identify $\mathcal{T}^{0}$ as the Lie algebra $\mathfrak{L}=L T^{0} \oplus \mathcal{S}_{n}(m) \oplus$ $\sum_{r \in \Gamma} \mathbb{C} t^{r} d_{0} \oplus \sum_{r \in \Gamma} \mathbb{C} t^{r} K_{0}$. Then $M$ is an irreducible $\mathfrak{L}$-module.

3.2. Let for $k \in \mathbb{Z}^{n}$ with $k=\left(k_{1}, \ldots, k_{n}\right)$, define $M_{0}^{\prime}=\bigoplus_{0 \leq k_{i}<m_{i}} M_{k}$, and $M_{r}^{\prime}=\bigoplus_{r_{i} \leq k_{i}<m_{i}+r_{i}} M_{k}$. Then $M=\bigoplus_{r \in \Gamma} M_{r}^{\prime}$ is $\Gamma$-graded. We have the following from [15].

Theorem 3.9. Consider $\mathfrak{L}$-irreducible module $M$ with $\Gamma$ grading. Then either $t^{s} K_{0}$ acts injectively on $M$ for all $0 \neq s \in \Gamma$ or $t^{s} K_{0}$ acts trivially on $M$ for all $0 \neq s \in \Gamma$.

Proof. For $D(u, r) \in \mathcal{S}_{n}(m)$, we have $\left[D(u, r), t^{s} K_{0}\right]=(u \mid s) t^{r+s} K_{0}$. As in our case $n \geq 2$, for $s \in \Gamma, s \neq 0$, we can always find $u \in \mathbb{C}^{n+1}$ and $r \in \mathbb{Z}^{n+1}$ with $r_{0}=0$, such that $(u \mid r)=0$, and $(u \mid s) \neq 0$. Now using the same argument as Proposition 3.4 of [15] we get the result. Note that the additional spaces $\mathrm{LT}^{0}$ and $\sum_{r \in \Gamma} \mathbb{C} t^{r} d_{0}$ don't create any problem as they commute with $t^{s} K_{0}$.

Proposition 3.10. Let $r, s \in \Gamma, k \in \mathbb{Z}^{n}$ and $0 \neq v \in M$. Then

(a) $t^{r} K_{0} v \neq 0$ for all $r \in \Gamma$.

(b) $t^{r} K_{0} t^{s} K_{0}=\lambda_{r, s} t^{r+s} K_{0}$ on $M$, where $\lambda_{r, s}=\lambda$ for all $r \neq 0, s \neq$ $0, r+s \neq 0, \lambda_{r,-r}=\mu$ for all $r \neq 0$ and $\lambda_{0, r}=C_{0}$ for all $r \in \Gamma$. Further we have $\mu C_{0}=\lambda^{2} \neq 0$.

(c) $\operatorname{dim} M_{k}=\operatorname{dim} M_{k+r}=p_{k} \forall r \in \Gamma$. Suppose $v_{1}(k), \ldots, v_{p_{k}}(k)$ is a basis for $M_{k}$, where $0 \leq k_{i}<m_{i}$. Let $v_{i}(k+s)=\frac{1}{\lambda} v_{i}(k), \forall i, \forall s \neq 0$. Then $v_{1}(k+s), \cdots, v_{p_{k}}(k+s)$ is a basis for $M_{k+s}$. 
INTEGRABLE MODULES FOR TWISTED TOROIDAL EXTENDED AFFINE LIE ALGEBRAS

(d) For $0 \neq r \in \Gamma, t^{r} K_{0}\left(v_{1}(k+s), \cdots, v_{p_{k}}(k+s)\right)=\lambda\left(v_{1}(k+s+\right.$ $\left.r), \cdots, v_{p_{k}}(k+s+r)\right)$.

(e) Recall that $\stackrel{\circ}{\mathfrak{g}}(\overline{0}, \overline{0})$ is a simple Lie algebra of fixed points with $\stackrel{\circ}{\mathfrak{h}}(0)$ be its Cartan subalgebra with $\alpha_{1}^{\vee}, \ldots, \alpha_{l}^{\vee}$ be its simple co-roots. There exists $\alpha \in \stackrel{\circ}{\mathfrak{h}}(0)^{*}$ such that $h(r)\left(v_{1}(k+s), \cdots, v_{p_{k}}(k+s)\right)=\alpha(h)\left(v_{1}(k+\right.$ $\left.s+r), \cdots, v_{p_{k}}(k+s+r)\right), \forall r \neq 0 ; \alpha\left(\alpha_{i}^{\vee}\right)=0$ iff $\lambda\left(\alpha_{i}^{\vee}\right)=0$.

(f) $t^{r} d_{0}\left(v_{1}(k+s), \cdots, v_{p_{k}}(k+s)\right)=\Lambda_{d}\left(v_{1}(k+s+r), \cdots, v_{p_{k}}(k+s+r)\right)$ for all $0 \neq r \in \Gamma, k \in \mathbb{Z}^{n}, \Lambda_{d} \in \mathbb{C}$ and $d_{0}(v(k))=\Lambda\left(d_{0}\right) v(k)$.

Proof. Let us assume that $t^{s} K_{0}=0$ on $M$ for all $0 \neq s \in \Gamma$. Then consider the highest root $\theta$ of $\stackrel{\circ}{\mathfrak{g}}(\overline{0}, \overline{0})$ and $x_{\theta}$ and $x_{-\theta}$ be elements of $\stackrel{\circ}{\mathfrak{g}}(\overline{0}, \overline{0})_{\theta}$ and $\stackrel{\circ}{\mathfrak{g}}(\overline{0}, \overline{0})_{-\theta}$ such that $\left\langle x_{\theta}, x_{-\theta}\right\rangle=1$. Then it is easy to see that $x_{-\theta} \otimes t_{0}$ and $x_{\theta} \otimes t_{0}^{-1}$ and $h_{\theta}+K_{0}$ form a $\mathfrak{s l}_{2}$ copy. Consider the Lie algebra $\mathfrak{s l}_{2} \otimes \mathbb{C}\left[t_{1}, t_{1}^{-1}\right]$ with bracket: $\left[\left(x_{-\theta} \otimes t_{0}\right) \otimes t_{1}^{r_{1}},\left(x_{\theta} \otimes t_{0}^{-1}\right) \otimes t_{1}^{s_{1}}\right]=\left(-h_{\theta}+K_{0}\right) \otimes t_{1}^{r_{1}+s_{1}}=$ $-h_{\theta} \otimes t_{1}^{r_{1}+s_{1}}+K_{0} \otimes t_{1}^{r_{1}+s_{1}}$.

Now let us look at the bracket in our setting $\left[x_{-\theta} \otimes t_{0} t_{1}^{r_{1}}, x_{\theta} \otimes t_{0}^{-1} t_{1}^{s_{1}}\right]=$ $-h_{\theta} \otimes t_{1}^{r_{1}+s_{1}}+t_{1}^{r_{1}+s_{1}} K_{0}+r_{1} t_{1}^{r_{1}+s_{1}} K_{1}$. But as $r_{1} t_{1}^{r_{1}+s_{1}} K_{1}$ acts trivially on $V$, we identify $K_{0} \otimes t_{1}^{r_{1}+s_{1}}$ with $t_{1}^{r_{1}+s_{1}} K_{0}$. Now as $V$ is an integrable $\mathcal{T}$ module for $\mathcal{T}$ in particular for $L\left(\mathfrak{s l}_{2}\right)=\mathfrak{s l}_{2} \otimes \mathbb{C}\left[t_{1}, t_{1}^{-1}\right]$ and every element of $M$ is highest weight vector for $L\left(\mathfrak{s l}_{2}\right)$. Now following the notations in [8]: for any positive $\operatorname{root} \beta$ of $\stackrel{\circ}{\mathfrak{g}}(\overline{0}, \overline{0}), \Lambda_{\beta}^{ \pm}(u)=\sum_{m=0}^{\infty} \Lambda_{\beta, \pm m} u^{m}=\exp \left(-\sum_{k=1}^{\infty} \frac{h_{\beta, \pm k}}{k} u^{k}\right)$, where $h_{\beta} \in \stackrel{\circ}{\mathfrak{h}}(0)$ and $h_{\beta, \pm k}=h_{\beta} \otimes t^{ \pm k}$. Let $\delta_{0}$ be the corresponding element of $\mathcal{H}^{*}$ with respect to $K_{0} \cdot \theta^{\prime}=-h_{\theta}+K_{0}$. So $K_{0}=-h_{\theta}+K_{0}+h_{\theta}$. Then by Proposition 1.1 of [8] we have $\Lambda_{\theta^{\prime}, m} v=0$ for all $m>\lambda\left(\theta^{\prime}\right)$. Similarly $\Lambda_{\theta, m} v=0$ for all $m>\lambda(\theta)$ and $\Lambda_{\theta^{\prime}, m} v \neq 0$ for $m=\lambda\left(\theta^{\prime}\right)$ and $\Lambda_{\theta, m} v \neq 0$ for $m=\lambda(\theta)$. But as $\Lambda_{\delta_{0}, m}=\sum_{m_{1}+m_{2}=m} \Lambda_{\theta^{\prime}, m_{1}} \Lambda_{\theta, m_{2}}$. From above it follows 
that $\Lambda_{\delta_{0}, \lambda\left(k_{0}\right)}=\Lambda_{\theta^{\prime}, \lambda\left(\theta^{\prime}\right)} \Lambda_{\theta, \lambda(\theta)}$. But as by assumption $t^{s} K_{0}=0$ on $M$ for all $0 \neq s \in \Gamma$. So $\Lambda_{K_{0}, m}=0$ for all $m>0$. But as RHS of the above equation is non-zero, this forces that $\lambda\left(K_{0}\right)=0$ which is a contradiction. So $t^{s} K_{0}$ acts injectively on $M$ for all $0 \neq s \in \Gamma$. By Theorem 3.9 we have $t^{s} K_{0} v \neq 0$, for any nonzero $v \in M$ and $\forall s \in \Gamma$ (for $0=s \in \Gamma, K_{0}$ acts by non-zero scalar $\left.C_{0}\right)$ and $\operatorname{dim} M_{k}=\operatorname{dim} M_{k+s}=p_{k}, \forall s \in \Gamma$. If $\left\{v_{1}, \ldots, v_{p_{k}}\right\}$ is a basis of $M_{k}$ then $\left\{v_{1}(k+s), \ldots, v_{p_{k}}(k+s)\right\}$ is a basis for $M_{k+s}$, where $\frac{1}{\lambda} t^{s} K_{0} v_{i}(k)=v(k+s)$ for all $0 \neq s \in \Gamma$ and $t^{s} K_{0}\left(v_{1}(k+r), \ldots, v_{p_{k}}(k+\right.$ $r))=\lambda\left(v_{1}(k+r+s), \ldots, v_{p_{k}}(k+r+s)\right), \forall s \in \Gamma, s \neq 0$. It follows that $M \cong\left(\bigoplus_{\substack{0 \leq k_{i}<m_{i} \\ 1 \leq i \leq n}} M_{k}\right) \otimes A(m)$. Let us denote $M^{1}=\bigoplus_{\substack{0 \leq k_{i}<m_{i} \\ 1 \leq i \leq n}} M_{k}$ and denote $v(0)=v$ for $v \in M^{1}$. Let $\frac{t^{r} K_{0}}{\lambda} v(0)=v(k), r \neq 0, r \in \Gamma$. Now (b) follows trivially. From (b) it follows that $\frac{t^{s}}{\lambda} v(r)=v(r+s)$. Now for fixed $i$, let $\alpha_{i}^{\vee}$ be a coroot of $\stackrel{\circ}{\mathfrak{h}}(0)$. As $\Omega_{A(m)} / d_{A(m)}$ acts trivially on $M$ by Proposition 3.8, $\stackrel{\circ}{\mathfrak{h}}(0) \otimes A(m)$ is abelian on $M$ and as $D(u, r) \alpha_{i}^{\vee}(s)=(u \mid s) \alpha_{i}^{\vee}(s+r)$, from [15] it follows that either $\alpha_{i}^{\vee}(s)$ is zero for all $s \neq 0, s \in \Gamma$ or $\alpha_{i}^{\vee}(s)$ is injective for all $s \neq 0, s \in \Gamma$. But as in previous case using [8] it follows that $\alpha_{i}^{\vee}$ acts trivially when $\alpha_{i}^{\vee}(s)$ acts trivially for all $0 \neq s \in \Gamma$. Hence we can assume that $\alpha_{i}^{\vee}(s)$ acts injectively for all $s \in \Gamma$. Now consider a nonzero scalar $b_{i} \in \mathbb{C}$. Then $D(u, r)\left(\alpha_{i}^{\vee}(s)-b_{i} t^{s} K_{0}\right)=(u \mid s)\left(\alpha_{i}^{\vee}(s)-b_{i} t^{s} K_{0}\right)$ for $u \in \mathbb{C}^{n}, r, s \in \Gamma, r, s \neq 0,(u \mid r)=0$. Then again by [15] it follows that $\alpha_{i}^{\vee}(s)-b_{i} t^{s} K_{0}$ is either zero or injective for all $s \in \Gamma$. Now for $0 \neq r \in \Gamma$, consider the nonzero operator $t^{-r} K_{0} \alpha_{i}^{\vee}(r)$ on $M_{0}$. Then there exists $0 \neq$ $v \in M_{0}$ such that $t^{-r} K_{0} \alpha_{i}^{\vee}(r) v=\lambda_{i} v$ for some $0 \neq \lambda_{i} \in \mathbb{C}$. Then operating $t^{r} K_{0}$ both side we get $\mu C_{0} \alpha_{i}^{\vee}(r) v=\lambda_{i} t^{r} K_{0} v$. Now since $\mu C_{0}=\lambda^{2} \neq 0$, we 
INTEGRABLE MODULES FOR TWISTED TOROIDAL EXTENDED AFFINE LIE ALGEBRA\$ get $\alpha_{i}^{\vee}(r) v-\frac{\lambda_{i}}{\mu C_{0}} t^{r} K_{0} v=0$ and so by earliar discussion we have $\alpha_{i}^{\vee}(r) v=$ $\frac{\lambda_{i}}{\mu C_{0}} t^{r} K_{0} v=\frac{\lambda_{i} \lambda}{\mu C_{0}} v(r)=\frac{\lambda_{i}}{\lambda} v(r)$ for all $0 \neq r \in \Gamma$. Now defining $\alpha: \stackrel{\circ}{\mathfrak{h}}(0) \mapsto \mathbb{C}$ by $\alpha\left(\alpha_{i}^{\vee}\right)=\frac{\lambda_{i}}{\lambda}$ for $1 \leq i \leq n$, we get the desired result. Similarly (f) follows as $\left[D(u, s), t^{r} d_{0}\right]=(u \mid r) t^{r+s} d_{0}$, hence by similar argument as (e), we get the desired result.

3.3. Now using $(d)$ of above proposition we identify $t^{r} K_{0}$ with $\frac{1}{\lambda} t^{r}$ for $r \in \Gamma$. Hence $M^{1} \otimes A(m)$ becomes an irreducible module for the space $\mathfrak{L}=L T^{0} \oplus \mathcal{S}_{n}(m) \oplus \sum_{r \in \Gamma} \mathbb{C} t^{r} d_{0} \oplus A(m)$. Now we will rewrite $L T^{0}$ for our convenience: for that let $\mathfrak{g}(0)=\left\{X \in \stackrel{\circ}{\mathfrak{g}} \mid \sigma_{0}(X)=X,[h, X]=0, h \in \stackrel{\circ}{\mathfrak{h}}(0)\right\}$. Then $\mathfrak{g}(0) \neq 0$ as $\stackrel{\circ}{\mathfrak{h}}(0) \subseteq \mathfrak{g}(0)$ and $\sigma_{i}(\mathfrak{g}(0)) \subseteq \mathfrak{g}(0)$. So $\mathfrak{g}(0)$ preserves $\Lambda$ grading and let $\mathfrak{g}(0)=\bigoplus_{\bar{k} \in \Lambda}(\mathfrak{g}(0))_{\bar{k}}$ and let $L(\mathfrak{g}(0), \sigma)=\bigoplus_{k \in Z^{n+1}}(\mathfrak{g}(0))_{\bar{k}} \otimes t^{k}$ be the corresponding multiloop algebra. Then it is easy to see that $L T^{0}=$ $L(\mathfrak{g}(0), \sigma)$. Now consider the subspace $W$ of $M=M^{1} \otimes A(m)$ spanned by $\left\{v(r)-v(0): v \in M^{1}, r \in \Gamma\right\}$. Then $W$ is module for $L(\mathfrak{g}(0), \sigma) \rtimes \mathcal{S}_{n}^{\prime}(m) \oplus$ $A(m) \oplus \sum_{r \in \Gamma} \mathbb{C} t^{r} d_{0}$, where $\mathcal{S}_{n}^{\prime}(m)=\operatorname{span}\left\{D(u, r)-D(u, 0): u \in \mathbb{C}^{n}, r \in\right.$ $\Gamma,(u \mid r)=0\}$. Let us consider $\widetilde{V}=\left(M^{1} \otimes A(m)\right) / W$. By previous argument $M / W$ is a finite dimensional $L(\mathfrak{g}(0), \sigma) \rtimes \mathcal{S}_{n}^{\prime}(m) \oplus A(m) \oplus \sum_{r \in \Gamma} \mathbb{C} t^{r} d_{0}$. As $A(m) \oplus \sum_{r \in \Gamma} \mathbb{C} t^{r} d_{0}$ act as scalars on $\widetilde{V}$, we neglect them and consider $\widetilde{V}$ as $\mathcal{I}:=L(\mathfrak{g}(0), \sigma) \rtimes \mathcal{S}_{n}^{\prime}(m)$-module. Now define $L\left(V^{\prime}\right)=V^{\prime} \otimes A_{n}$, where $V^{\prime}$ is any $\mathcal{I}$ module. Let fix $\gamma \in P(V)$, where $V$ is an integrable irreducible module for $\mathcal{T}$ with finite dimensional weight spaces. Let $\beta_{i}=\gamma\left(d_{i}\right)$ for $1 \leq i \leq n$, let $\beta=\left(\beta_{1}, \ldots \beta_{n}\right) \in \mathbb{C}^{n}$. Let $\mu \in \mathbb{C}^{n}$ be arbitrary. Let $V^{\prime}$ be any 
$\mathcal{I}$-module. Then $L\left(V^{\prime}\right)$ is a $\mathfrak{L}$-module by the following action:

$$
\begin{aligned}
X(k) \cdot v \otimes t^{s} & =(X(k) v) \otimes t^{k+s} ; \\
0 \neq r \in \Gamma, D(u, r) \cdot\left(v \otimes t^{s}\right) & =(I(u, r) v) \otimes t^{r+s}+(u \mid s+\mu) v \otimes t^{s+r} \\
t^{l} \cdot v \otimes t^{s} & =v \otimes t^{s+l} ; \\
t^{l} d_{0} \cdot v \otimes t^{s} & =\gamma\left(d_{0}\right) \cdot v \otimes t^{t+s} \\
D(u, 0) v \otimes t^{l} & =(u \mid \beta) v \otimes t^{l} \\
\frac{t^{r}}{\lambda} K_{0} \cdot v \otimes t^{l} & =v \otimes t^{r+l}, r \neq 0, r \in \Gamma \\
\frac{K_{0}}{C_{0}} v \otimes t^{l} & =v \otimes t^{l} .
\end{aligned}
$$

where $v \in V, s, l \in \Gamma, u \in \mathbb{C}^{n}, r \in \Gamma$ with $(u \mid r)=0$.

It is easy to check that $L\left(V^{\prime}\right)$ is an $\mathfrak{L}$-module (see 8.3 of [2]). Now we take $\widetilde{V}=M / W$. Then by above $L(\widetilde{V})$ is an $\mathfrak{L}$-module. Consider a map $\widetilde{\phi}: M \mapsto L(\widetilde{V})$ by $\widetilde{\phi}\left(v_{k}\right)=\bar{v}_{k} \otimes t^{k}, k \in \mathbb{Z}^{n}$, where $v_{k} \in M_{k}, \bar{v}_{k} \in M / W$. It follows that $\widetilde{\phi}$ is a non-zero $\mathfrak{L}$-module map. Since $M$ is an irreducible $\mathfrak{L}$-module, it follows that $\widetilde{\phi}$ is injective. In particular $\widetilde{\phi}(M) \subseteq L(\widetilde{V})$. Let us define for a fixed $p \in \Lambda$. $L(\widetilde{V})(\bar{p})=\left\{\bar{v}_{k} \otimes t^{k+r+p}, r \in \Gamma, k \in \mathbb{Z}^{n}\right\}$. Then $L(\widetilde{V})=\bigoplus_{\bar{p} \in \Lambda} L(\widetilde{V})(\bar{p})$ Then $M \cong L(\widetilde{V})(\overline{0})$. In particular $L(\widetilde{V})(\overline{0})$ is an irreducible $\mathfrak{L}$-module (see section 8 of [2] for details).

\section{4 .}

4.1. In order to get hold of $M$, by previous section, we need to understand $\widetilde{V}$ as $\mathcal{I}$-module. We devote this section for this purpose. Let $\mathfrak{g}(0)=\mathfrak{g}(0)_{\text {ss }} \oplus$ $R$, where $\mathfrak{g}(0)_{\mathrm{ss}}$ and $R$ are Levi and radical part of $\mathfrak{g}(0)$. Then as $\sigma_{i}\left(\mathfrak{g}(0)_{\mathrm{Ss}}\right)=$ 
INTEGRABLE MODULES FOR TWISTED TOROIDAL EXTENDED AFFINE LIE ALGEBRAg

$\mathfrak{g}(0)_{\mathrm{ss}}$ and $\sigma_{i}(R)=R$ for $1 \leq i \leq n$, we have $L(\mathfrak{g}(0), \sigma)=L\left(\mathfrak{g}(0)_{\mathrm{ss}}, \sigma\right) \oplus$ $L(R, \sigma)$. Now we invoke result from [2]

Theorem 4.1. $\widetilde{V}$ is completely reducible as $\mathcal{I}$ module with isomorphic irreducible components.

Proof. The proof of this theorem is exactly similar to Theorem 8.3 of [2].

In the light of above proposition it is enough to consider an irreducible representation of $\mathcal{I}$. Let $(W, \pi)$ be a finite dimensional irreducible representation of $L(\mathfrak{g}(0), \sigma) \rtimes \mathcal{S}_{n}^{\prime}(m)=\mathcal{I}$. Let $\pi(L(\mathfrak{g}(0), \sigma))=\mathfrak{g}^{\prime \prime}$. As $\pi(\mathcal{I})$ is reductive with atmost one dimensional center, there exists a unique compliment $\mathfrak{g}$ in $\pi(\mathcal{I})$. So $W$ is an irreducible module for $\mathfrak{g}^{\prime} \oplus \mathfrak{g}^{\prime \prime}$. Then it is standard that $W \cong W_{1} \otimes W_{2}$, where $W_{1}$ and $W_{2}$ are irreducible modules for $\mathfrak{g}^{\prime}$ and $\mathfrak{g}^{\prime \prime}$ respectively. Now we concentrate on $W_{2}$ as $L(\mathfrak{g}(0), \sigma)=L\left(\mathfrak{g}(0)_{\mathrm{ss}}, \sigma\right) \oplus L(R, \sigma)$. Now as $R$ is solvable ideal, it follows that $\pi(L(R, \sigma))$ lies in the center of $\pi(\mathcal{I})$ which is atmost one dimensional. Hence $L(R, \sigma)$ acts as a scalar on $W$. In particular $V_{2}$ is an irreducible module for $L\left(\mathfrak{g}(0)_{\mathrm{ss}}, \sigma\right)$. Now we recall result from [22] for which we need to introduce some notations: For each $i, 1 \leq i \leq n$, and a fixed positive integer $l$, let $\underline{a}_{i}=\left(a_{i, 1}, a_{i, 2}, \cdots, a_{i, l}\right)$ such that $a_{i, j}^{m_{i}} \neq a_{i, t}^{m i}$ for $j \neq t\left({ }^{* *}\right)$. Let $\overline{\mathfrak{g}}$ be a finite dimensional semisimple Lie algebra. Let $\sigma_{1}, \sigma_{2}, \cdots, \sigma_{n}$ be finite order automorphisms on $\overline{\mathfrak{g}}$ of order $m_{1}, m_{2}, \cdots m_{n}$ respectively. Let $L(\overline{\mathfrak{g}}, \sigma)$ be corresponding multiloop algebra. Let $I=\left\{\left(i_{1}, i_{2}, \ldots, i_{n}\right) \mid 1 \leq i_{j} \leq l\right\}$. Let for $S \in I$, $S=\left(i_{1}, i_{2}, \ldots, i_{n}\right)$ and $r=\left(r_{1}, r_{2}, \cdots, r_{n}\right) \in \mathbb{Z}^{n}, a_{S}^{r}=a_{1, i_{1}}^{r_{1}} a_{2, i_{2}}^{r_{2}} \cdots a_{n, i_{n}}^{r_{n}}$. Now consider the evaluation map $\phi: \overline{\mathfrak{g}} \otimes A \mapsto \bigoplus \overline{\mathfrak{g}}(n l$ copies $), \pi(X \otimes$ $\left.\left.\left.t^{r}\right)=\left(a_{I_{1}}^{r}(X), a_{I_{2}}^{r}(X)\right), \ldots, a_{I_{n l}}^{r}(X)\right)\right)$, where $I_{1}, I_{2}, \ldots, I_{n l}$ is some order on 
I. Consider restriction of $\phi$ to $L(\overline{\mathfrak{g}}, \sigma)$. We have the following theorem which follows from [22]:

Theorem 4.2. Let $W^{\prime}$ be a finite dimensional irreducible representation of $L(\overline{\mathfrak{g}}, \sigma)$. Then represention factors though $\bigoplus \overline{\mathfrak{g}}($ nl copies $)$.

Proof. See 22] Corollary 6.1 and the discussion thereafter. Note that some factors in $\bigoplus \overline{\mathfrak{g}}(n l$ copies $)$ may act trivially and we discard them.

From above result it follows that $\bigoplus \mathfrak{g}(0)_{\mathrm{ss}} \cong \mathfrak{g}_{\mathrm{sS}}^{\prime \prime}$ as both can realised as the quotient $L\left(\mathfrak{g}(0)_{\mathrm{ss}}, \sigma\right) / \operatorname{Ker}\left(\pi_{1}\right)$. So we have $\pi\left(L(\mathfrak{g}(0), \sigma) \rtimes \mathcal{S}_{n}^{\prime}(m)\right)=$ $\oplus \mathfrak{g}(0)_{\text {ss }} \bigoplus \mathbb{C} \bigoplus \mathfrak{g}^{\prime \prime}$. Now we choose $i$-th piece of $\bigoplus \mathfrak{g}(0)_{\text {ss }}$ and choose projection of the map $\pi$, say $\pi_{i}$ onto it, i.e., $\pi_{i}\left(L(\mathfrak{g}(0), \sigma) \rtimes \mathcal{S}_{n}^{\prime}(m)\right) \mapsto \mathfrak{g}(0)_{\mathrm{ss}}$. Then we claim that $\pi_{i}\left(S_{n}^{\prime}(m)\right)=\{0\}$. For consider $[D(u, r)-D(u, 0), X(k+s)]=$ $(u \mid k+s) X(k+s+r)-X(k+s)$, where $k \in \mathbb{Z}^{n}, s, r \in \Gamma$. Apply $\pi_{i}$ both side we get $\left[\pi_{i}(D(u, r)-D(u, 0)), a_{I_{i}}^{k+s} X\right]=(u \mid k+s) a_{I_{i}}^{k+s}(X)\left(a_{I_{i}}^{r}-1\right)$. Now cancelling $a_{I_{i}}^{k+s}$ both side we get $\left[\pi_{i}(D(u, r)-D(u, 0)), X\right]=(u \mid k+s)\left(a_{I_{i}}^{r}-1\right)(X)$. Now as LHS is independent of $s$, this forces that $\pi_{i}(D(u, r)-D(u, 0))=$ 0. We deduce that $a_{I_{i}}^{r}=1$ for all $r \in \Gamma$. From this it follows that $\pi_{i}(X(k+s)-X(k))=0$. As these equations take place in semisimple Lie algebra which has no center, hence the claim follows. Now in view of condition $\left({ }^{* *}\right)$ we see that the representation of $L\left(\mathfrak{g}(0)_{\mathrm{ss}}, \sigma\right)$ factors through only one copy of $L\left(\mathfrak{g}(0)_{\mathrm{ss}}\right.$, i.e., $\mathfrak{g}(0)_{\mathrm{ss}} \cong \mathfrak{g}_{\mathrm{ss}}^{\prime}$.

4.2. Now we turn our attention towards finite dimensional irreducible modules for $\mathcal{S}_{n}^{\prime}$. For clarity we once again recall the notations: $A_{n}=A=$ $\mathbb{C}\left[t_{1}^{ \pm 1}, \cdots, t_{n}^{ \pm 1}\right]$, and $\mathcal{S}_{n}=\left\{D(u, r): u \in \mathbb{C}^{n}, r \in \mathbb{Z}^{n},(u \mid r)=0\right\}$, where $D(u, r)=\sum_{i=1}^{n} u_{i} t^{r} t_{i} \frac{d}{d t_{i}}$, where $u=\left(u_{1}, \ldots, u_{n}\right) \in \mathbb{C}^{n}$ and $r=\left(r_{1}, \ldots, r_{n}\right) \in$ 
INTEGRABLE MODULES FOR TWISTED TOROIDAL EXTENDED AFFINE LIE ALGEBRA\$

$\mathbb{Z}^{n}$ and $\mathcal{S}_{n}^{\prime}=\operatorname{span}\left\{D(u, r)-D(u, 0) \mid u \in \mathbb{C}^{n}, r \in \mathbb{Z}^{n},(u \mid r)=0\right\}$. Then $\mathcal{S}_{n}^{\prime}$ is a Lie algebra with brackets: $[I(v, s), I(u, r)]=(u, s) I(v, s)-(v, r) I(u, r)+$ $I(w, r+s)$ where $w=(v, r) u-(u, s) v, v, u \in \mathbb{C}^{n}, r, s \in \mathbb{Z}^{n}$ and $I(u, r)=$ $D(u, r)-D(u, 0)$. Now consider the Lie algebra $\mathcal{S}_{n} \ltimes A$. Our aim is try to understand the relation between finite dimensional $\mathcal{S}_{n}^{\prime}$ module and modules with $\mathcal{S}_{n} \ltimes A$ with finite dimensional weight spaces. For $\mathcal{S}_{n} \ltimes A$, the subalgebra $\left\{d_{i}=t_{i} \frac{d}{d t}: 1 \leq i \leq n\right\}$ plays the role of a Cartan subalgebra. A module for $\mathcal{S}_{n} \ltimes A$ we mean $A$ action is associative. In particular $t^{0}=1$ acts as identity. The action of $t^{r}$ is invertible for $r \in \mathbb{Z}^{n}$. We recall a Theorem of [5]. Let $\tilde{V}$ be an irreducible module for $\mathcal{S}_{n} \otimes A$ with finite dimensional weight spaces. Let $W^{\prime}$ be an $\mathfrak{s l}_{n}$ module and extend to $\mathfrak{g l}_{n}$ by letting $I$ act trivially. Let $E_{a b}$ be generators for $\mathfrak{g l}_{n}$. Let $I=\sum_{i=1}^{n} E_{a a}$. Note: Suppose $I(v, s)$ is replaced by $I(v, s)+(v, \gamma)$ for some $\gamma \in \mathbb{C}^{n}$. Then these elements also satisfy above bracket. We make the following observations:

(1) Suppose $W$ is a finite dimensional module for $\mathcal{S}_{n}^{\prime}$. Then $\left(\pi_{\alpha, \beta}, W \otimes\right.$ $A=L(W))$ is a $\mathcal{S}_{n} \ltimes A$ module in the following way:

for $r \neq 0, D(u, r)\left(w \otimes t^{k}\right)=(I(u, r) w) \otimes t^{r+k}+(u \mid \beta+k) w \otimes t^{r+k}$; $D(u, 0)\left(w \otimes t^{k}\right)=(u \mid \alpha+k) w \otimes t^{k}$.

(2) Suppose $V=\bigoplus_{k \in \mathbb{Z}^{n}} V_{\alpha+k}$ is an weight module for $S_{n} \ltimes A$ such that $D(u, 0)\left(w \otimes t^{k}\right)=(u \mid \alpha+k) w \otimes t^{k}, v \in V_{\alpha+k}$. Put $t^{r} V_{\alpha}=V_{\alpha+r}$. Then $V=V_{\alpha} \otimes A . \quad D(u, 0) v \otimes t^{k}=(u \mid \alpha+k) v \otimes t^{k}, v \in V_{\alpha}$. Let $W=\left\{v \otimes t^{k}-v(0) \mid v \in V_{\alpha}\right\}$. It can be checked to be $\mathcal{S}_{n}^{\prime}$-module. Now consider a $\mathcal{S}_{n}^{\prime}$-module $\bar{V}=\frac{V_{\alpha} \otimes A}{W}$. Let us denote it by $(\theta, \bar{V})$. Define $\left(\theta_{\gamma}, \bar{V}\right) \mathcal{S}_{n}^{\prime}$-module $\left(\theta_{\gamma} I(u, r)\right) v=\left(\theta(I(u, r)) v+(u, \gamma) v\right.$ for $\gamma \in \mathbb{C}^{n}$ Thus $\theta_{0}=\theta$. 
The following can be checked:

(a) Suppose $(\theta, W)$ is a $\mathcal{S}_{n}^{\prime}$-module., where $W$ is finite dimensional. Let $\left(\pi_{\alpha, \beta}, L(W)\right)$ is a $\mathcal{S}_{n} \ltimes A$-module. Then $\left(\theta_{\alpha-\beta}, \overline{L(W)}\right) \cong$ $(\theta, W)$ as $\mathcal{S}_{n}^{\prime}$-module.

(b) Suppose $(\pi, W \otimes A)$ is $\mathcal{S}_{n} \ltimes A$-module with $\operatorname{dim} W<\infty$ with $D(u, 0) v(k)=(u \mid \alpha+k) v(k)$. Let $\left(\theta_{\mu}, \overline{W \otimes A}\right)$ be $\mathcal{S}_{n}^{\prime}$ - module. Then $\left(\pi_{\alpha, \alpha-\mu}, L(\overline{W \otimes A})\right) \cong(\pi, W \otimes A)$ as $\mathcal{S}_{n} \ltimes A$-module.

The following are easy to prove:

Lemma 4.3. Suppose $(\theta, W)$ is a finite dimensional irreducible $\mathcal{S}_{n}^{\prime}$-module. Then $\left(\pi_{\alpha, \beta}, L(W)\right)$ is an irreducible $\mathcal{S}_{n} \ltimes A$-module.

Lemma 4.4. Suppose $\left(\pi, L(W)\right.$ is an irreducible $\mathcal{S}_{n} \ltimes A$-module with dim $W<\infty$. Then $\left(\theta_{r}, \overline{L(W)}\right)$ is an irreducible finite dimensional module for $\mathcal{S}_{n}^{\prime}$

Theorem 4.5. Let $W$ be finite dimensional irreducible module for $\mathfrak{s l}_{n}$ and extend it to $\mathfrak{g l}_{n}$ by letting I trivially. Let $E_{i j}$ be generators of $\mathfrak{g l}_{n}$. Then $W$ can be made into $S_{n}^{\prime}$-module by the action: $I(u, r) w=\sum_{i, j} u_{i} r_{j} E_{j i} w+$ $(u, \gamma) w$, where $\gamma \in \mathbb{C}^{n}$.

Then $W$ is irreducible as $\mathcal{S}_{n}^{\prime}$-module, as $\left(\pi_{\alpha, \beta}, L(W)\right)$ is an ireducible $\mathcal{S}_{n} \ltimes A$ for any $\alpha, \beta \in \mathbb{C}^{n}$. We will now recall a result from [5] where they have classified irreductible $S_{n} \ltimes A$ modules with finite dimensional weight spaces. Using this it follows that all irreducible finite dimensional $S_{n}^{\prime}$-modules occur as in Theorem 4.5 . 
INTEGRABLE MODULES FOR TWISTED TOROIDAL EXTENDED AFFINE LIE ALGEBRAs

Theorem 4.6. Suppose $W$ is a finite dimensional irreducible $\mathfrak{s l}_{n}$-module (extend $W$ to $\mathfrak{g l}_{n}$ trivially as before). Let $\alpha, \beta \in \mathbb{C}^{n}$. Consider $W \otimes A$ and the action $D(u, r)\left(w \otimes t^{k}\right)=(u, k+\beta) w \otimes t^{k+r}+\sum_{i, j} u_{i} r_{j} E_{j i} w \otimes t^{k+r}$, for $r \neq 0$ and $D(u, 0)\left(w \otimes t^{k}\right)=(u, \alpha+k) w \otimes t^{k}$ and $t^{r}\left(w \otimes t^{k}\right)=w \otimes t^{k+r}$. And all irreducible representations of $S_{n} \ltimes A$ module with finite dimensional weight spaces occur in this way.

We summerise the results of this section. We have proved that $\pi\left(S_{n}^{\prime}\right) \supseteq \mathfrak{g}^{\prime}$ and $\mathfrak{g}^{\prime}=\mathfrak{s l}_{n}(\mathbb{C})$. So $\pi(\widetilde{L})=\mathfrak{s l}_{n}(\mathbb{C}) \oplus \mathfrak{g}_{\mathrm{ss}}^{\circ} \oplus \mathbb{C} c$. Now take a finite dimensional irreducible module for $\mathfrak{s l}_{n}(\mathbb{C})$. Note that $\mathfrak{g}_{\mathrm{ss}}$ is $\Lambda$-graded. Let $W_{2}$ be finite dimensional $\Lambda$-graded irreducible module for $\mathfrak{g}_{\mathrm{ss}}^{\circ}$ such that it is compatible with respect to $\Lambda$ - grading of $\mathfrak{g}_{\mathrm{ss}}^{\circ}$ (in reality the gradation is given by the quotient of $\Lambda$ but we assume it is $\Lambda$-graded to avoid additional notations; see section 8 of [2]). Recall that $\widetilde{V}=\bigoplus M_{i}$, where all $M_{i}$ 's are isomorphic as $\widetilde{L}$-modules. Each $M_{i}$ is isomorphic to $V^{\prime} \otimes V^{\prime \prime}$ as $\mathfrak{s l}_{n} \oplus \mathfrak{g}_{\mathrm{ss}}^{\circ}$ module. Then $\widetilde{V}=V^{\prime} \otimes \sum V^{\prime \prime}$.

\section{Description of MOdules For $\mathcal{T}$}

Recall that $\mathcal{T}^{0}=L(\stackrel{\circ}{\mathfrak{g}}(0), \sigma) \oplus Z^{0} \oplus \mathcal{S}_{n+1}^{0}\left(m_{0}, m\right)$, where $Z^{0}=\bigoplus_{0 \leq i \leq n, s \in \Gamma} \mathbb{C} t^{s} K_{i}$ and $\mathcal{S}_{n+1}^{0}\left(m_{0}, m\right)$ is identified with $\mathcal{S}_{n}(m) \oplus \sum_{r \in \Gamma} \mathbb{C} t^{r} d_{0}$. Now let, $W_{1}$ and $W_{2}$ be irreducible modules for $\mathfrak{s l}_{n}$ extended trivially to $\mathfrak{g l}_{n}$ and $\mathfrak{g}(0)$ respectively with $W_{2}$ being $\Lambda$-graded which is compatible with $\Lambda$-gradation of $\mathfrak{g}(0)$. Define $\mathcal{T}^{0}$ action on $W_{1} \otimes W_{2} \otimes A_{n}$ as follows: For $\alpha, \beta \in \mathbb{C}^{n}, r \neq 0, r \in \Gamma, \gamma \in$

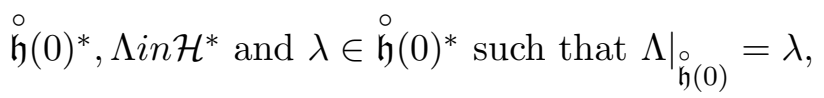
$D(u, r)\left(w_{1} \otimes w_{2} \otimes t^{k}\right)=(u, k+\beta) w_{1} \otimes w_{2} \otimes t^{k+r}+\left(\sum_{i, j} u_{i} r_{j} E_{j i} w_{1}\right) \otimes w_{2} \otimes t^{k+r}$. For $r=0, D(u, 0)\left(w_{1} \otimes w_{2} \otimes t^{k}\right)=(u, k+\alpha) w_{1} \otimes w_{2} \otimes t^{k}$. 
$X \in \stackrel{\circ}{\mathfrak{g} s}, \bar{k}, X \otimes t^{k}\left(w_{1} \otimes w_{2} \otimes t^{l}\right)=w_{1} \otimes X w_{2} \otimes t^{k+l}, k, l \in \mathbb{Z}^{n}$.

$r \neq 0, r \in \Gamma, \frac{1}{\lambda} t^{r} K_{0}\left(w_{1} \otimes w_{2} \otimes t^{l}\right)=w_{1} \otimes w_{2} \otimes t^{k+l} ;$

$K_{0}\left(w_{1} \otimes w_{2} \otimes t^{k}\right)=C_{0}\left(w_{1} \otimes w_{2} \otimes t^{k}\right)$.

For $r \in \Gamma, r \neq 0, d_{0} \otimes t^{r}\left(w_{1} \otimes w_{2} \otimes t^{l}\right)=\Lambda_{d}\left(w_{1} \otimes w_{2} \otimes t^{l+r}\right)$, where $\Lambda_{d} \in \mathbb{C}$; $r=0, d_{0}\left(w_{1} \otimes w_{2} \otimes t^{l}\right)=\Lambda\left(d_{0}\right) w_{1} \otimes w_{2} \otimes t^{l}$.

$r \neq 0, h \in \mathfrak{h}(0), h \otimes t^{r}\left(w_{1} \otimes w_{2} \otimes t^{l}\right)=\gamma(h)\left(w_{1} \otimes w_{2} \otimes t^{l+r}\right), r=0, h\left(w_{1} \otimes w_{2} \otimes\right.$ $\left.t^{l}\right)=\lambda(h) w_{1} \otimes w_{2} \otimes t^{l} ; \lambda(h)=0$ iff $\gamma(h)=0$. Now take a one dimensional representation of $L(R, \sigma)$ say $\psi$. Then $L(R, \sigma)$ acts as a $\psi$. More precisely for $y \otimes t^{k}, y \in R_{\bar{k}}$, Then $y \otimes t^{k}\left(w_{1} \otimes w_{2} \otimes t^{l}\right)=\psi(y)\left(w_{1} \otimes w_{2} \otimes t^{k+l}\right)$. One can check that $W_{1} \otimes W_{2} \otimes A_{n}$ is $\mathfrak{g}(0) \otimes A_{n} \oplus Z^{0} \oplus \mathcal{S}_{n+1}^{0}\left(m_{0}, m\right)$. It has $\mathcal{T}^{0}$ - module structure by considering $\Lambda$ gradation of $W_{2}=\bigoplus_{\bar{k} \in \Lambda} W_{2, \bar{k}}$ which is compatible with $\Lambda$-gradation of $\mathfrak{g}(0)$. Then it follows that the submodule $M^{\prime}=\bigoplus_{k \in \mathbb{Z}^{n}} W_{1} \otimes W_{2, \bar{k}} \otimes t^{k}$ is an irreducible module for $\mathcal{T}^{0}$. Consider the induced module $\mathcal{M}=\operatorname{Ind}_{\mathcal{T}^{0} \oplus \mathcal{T}+} M^{\prime}$, with $\mathcal{T}^{+}$acting trivially on $M^{\prime}$. Let $\mathcal{M}^{\mathrm{rad}}$ be the unique maximal submodule of $\mathcal{M}$. Then $\mathcal{M} / \mathcal{M}^{\mathrm{rad}}$ is an ireducible module for $\mathcal{T}$. We have the following:

Theorem 5.1. Let $V$ be an irreducible integrable $\mathcal{T}$ module with finite dimensional weight spaces, with $K_{0}$ acting as $C_{0} \in \mathbb{Z}_{>0}$ and rest of $K_{i}$ 's act trivially for $1 \leq i \leq n$. Then $V \cong \mathcal{M} / \mathcal{M}^{\mathrm{rad}}$.

Remark 5.2. Using $\Lambda$-gradation on $\widetilde{V}$ one can define $\Lambda$-gradation on $L(\widetilde{V})$. In case if $\widetilde{V}$ is irreducible as $\mathcal{I}$-module, then it follows that $M$ is isomorphic as $\mathcal{T}^{0}$-module to the zeroth graded piece of $L(\widetilde{V})$ with respect to $\Lambda$-gradation. When $\widetilde{V}$ is reducible as $\mathcal{I}$-module, inclusion of $M$ in $L(\widetilde{V})$ is more complex. 
INTEGRABLE MODULES FOR TWISTED TOROIDAL EXTENDED AFFINE LIE ALGEBRAS

Infact there are several copies of $M$ inside $L(\widetilde{V})$. See Section 8 of [2] for more details.

\section{References}

[1] Bruce Allison, Stephen Berman, John Faulkner, and Arturo Pianzola. Multiloop realization of extended affine Lie algebras and Lie tori. Trans. Amer. Math. Soc., 361(9):4807-4842, 2009.

[2] Punita Batra and Senapathi Eswara Rao. On integrable modules for the twisted full toroidal Lie algebra. J. Lie Theory, 28(1):79-105, 2018.

[3] Yuly Billig. Representations of toroidal extended affine Lie algebras. J. Algebra, 308(1):252-269, 2007.

[4] Yuly Billig and Michael Lau. Irreducible modules for extended affine Lie algebras. J. Algebra, 327:208-235, 2011.

[5] Yuly Billig and John Talboom. Classification of category $\mathcal{J}$ modules for divergence zero vector fields on a torus. J. Algebra, 500:498-516, 2018.

[6] Vyjayanthi Chari, Ghislain Fourier, and Tanusree Khandai. A categorical approach to Weyl modules. Transform. Groups, 15(3):517-549, 2010.

[7] Vyjayanthi Chari and Jacob Greenstein. Graded level zero integrable representations of affine Lie algebras. Trans. Amer. Math. Soc., 360(6):2923-2940, 2008.

[8] Vyjayanthi Chari and Andrew Pressley. Weyl modules for classical and quantum affine algebras. Represent. Theory, 5:191-223, 2001.

[9] Fulin Chen, Zhiqiang Li, and Shaobin Tan. Classification of integrable representation for toroidal extended affine lie algebras. arXiv:1803.01324.

[10] Fulin Chen, Zhiqiang Li, and Shaobin Tan. Integrable representations for toroidal extended affine Lie algebras. J. Algebra, 519:228-252, 2019.

[11] S. Eswara Rao. Classification of irreducible integrable modules for toroidal Lie algebras with finite dimensional weight spaces. J. Algebra, 277(1):318-348, 2004.

[12] S. Eswara Rao. Partial classification of modules for Lie algebra of diffeomorphisms of d-dimensional torus. J. Math. Phys., 45(8):3322-3333, 2004. 
[13] S. Eswara Rao. Irreducible representations for toroidal Lie-algebras. J. Pure Appl. Algebra, 202(1-3):102-117, 2005.

[14] S. Eswara Rao and Cuipo Jiang. Classification of irreducible integrable representations for the full toroidal Lie algebras. J. Pure Appl. Algebra, 200(1-2):71-85, 2005.

[15] Xiangqian Guo and Genqiang Liu. Jet modules for the centerless Virasoro-like algebra. J. Algebra Appl., 18(1):1950002, 24, 2019.

[16] James E. Humphreys. Introduction to Lie algebras and representation theory, volume 9 of Graduate Texts in Mathematics. Springer-Verlag, New York-Berlin, 1978. Second printing, revised.

[17] James E. Humphreys. Introduction to Lie algebras and representation theory, volume 9 of Graduate Texts in Mathematics. Springer-Verlag, New York, 1978. Second printing, revised.

[18] Cuipo Jiang and Daoji Meng. Integrable representations for generalized Virasorotoroidal Lie algebras. J. Algebra, 270(1):307-334, 2003.

[19] Haisheng Li. On certain categories of modules for affine Lie algebras. Math. Z., 248(3):635-664, 2004.

[20] Katsuyuki Naoi. Multiloop Lie algebras and the construction of extended affine Lie algebras. J. Algebra, 323(8):2103-2129, 2010.

[21] Erhard Neher. Extended affine Lie algebras. C. R. Math. Acad. Sci. Soc. R. Can., 26(3):90-96, 2004.

[22] Erhard Neher, Alistair Savage, and Prasad Senesi. Irreducible finite-dimensional representations of equivariant map algebras. Trans. Amer. Math. Soc., 364(5):2619-2646, 2012.

[23] Chengkang Xu. Partial classification of modules for the algebra of skew-derivations over the $d$-dimensional torus. J. Math. Phys., 57(10):101702, 10, 2016.

[24] Yoji Yoshii. Lie tori-a simple characterization of extended affine Lie algebras. Publ. Res. Inst. Math. Sci., 42(3):739-762, 2006. 
INTEGRABLE MODULES FOR TWISTED TOROIDAL EXTENDED AFFINE LIE ALGEBRA\$

School of mathematics, Tata Institute of Fundamental Research, Homi Bhabha Road, Mumbai 400005, India.

email: sena98672@gmail.com, senapati@math.tifr.res.in

Department of mathematics and statistics, IIT Kanpur, Kalyanpur, Kanpur, 208016, India.

sachinsh@iitk.ac.in

Harish-Chandra Research Institute, HBNI, Allahabad 211019, India. batra@hri.res.in 